\title{
HI and optical studies of Wolf-Rayet galaxies
}

\section{A. Omar and S. Jaiswal}

Aryabhatta Research Institute of observational sciences, Manora Peak, Nainital 263 129, India. email: aomar@aries.res.in

\begin{abstract}
The tidal interactions between galaxies are believed to be the main mechanism responsible for Wolf-Rayet (WR) phases in galaxies. The HI $21 \mathrm{~cm}$-line is the best tracer for detecting tidal features in galaxies. We are studying WR galaxies in the HI line using GMRT and in the $\mathrm{H} \alpha$ line using Devasthal Optical telescope. Signatures of tidal interactions in Mrk 996 and Mrk 005, previously believed to be in non-interacting environment, have been detected using both $\mathrm{HI}$ and $\mathrm{H} \alpha$ images.
\end{abstract}

Keywords. galaxies: dwarf — radio lines: galaxies — galaxies: ISM

\section{Introduction, Galaxy Sample and Main Results}

Wolf-Rayet (WR) galaxies are characterized by presence of broad emission lines of He, $\mathrm{C}, \mathrm{N}$, and $\mathrm{O}$, which originate in the stellar winds of hot WR stars. The WR stars are the most massive evolved $\mathrm{O} / \mathrm{B}$ type stars with masses $\left(\mathrm{M}>20 \mathrm{M}_{\odot}\right)$ and therefore have very short life ( $\sim$ a few Myr). Due to this short WR phase in galaxies, the onset/triggering mechanisms of massive star formation can be best studied through WR galaxies.

Tidal interactions between galaxies have been confirmed by various observations and simulations, to be the main cause for onset of vigorous star formation in galaxies in low galaxy-density environments (e.g., in groups or fields). Most of the WR galaxies detected in SDSS show disturbances in their optical/NIR images. However, a few do not. We are studying several isolated WR galaxies in the HI $21 \mathrm{~cm}$-line using the Giant Meterwave Radio telescope (GMRT) as the HI line is the best tracer for interactions between galaxies. Additionally, H-alpha imaging and visible range spectroscopy are being performed using 1.3 meter Devasthal optical telescope and 2-m IUCAA telescope.

The galaxies are selected primarily from SDSS. As part of this study, 22 galaxies including several dwarf WR galaxies (Mrk 996, Mrk 475, Mrk 22, ISZ 59, UM 311, UM 439, UGCA 116, Mrk 5, CGCG 038-051, SBS 1222+614, UGC 9273, IC 2828, IC 2524, KUG 1013+381, NGC 1087, NGC 4398, NGC 3949, NGC 941, IC 3521, NGC 3381, IC 745 , NGC 5147) have been observed.

In Mrk 996, HI bridge and a low mass companion galaxy in the line of sight of Mrk 996 have been detected. Additionally, the $\mathrm{H} \alpha$ disk is found to be misaligned with the old stellar disk. No radio continuum is detected implying that either there is a lack of magnetic field or supernova explosions from the current phase of star-burst has not taken place in the galaxy. Similarly, In Mrk 5, HI bridge with a nearby HI cloud has been detected. These results provided first direct evidence of tidal interaction in Mrk 996 and Mrk 5. This survey will provide a conclusive statement on triggering mechanisms of star formation in WR galaxies. 